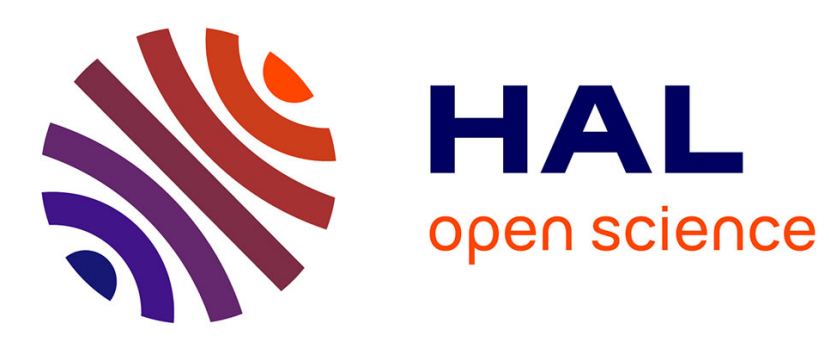

\title{
Statics and dynamics near the nematic-columnar phase transition in liquid crystals
}

J. Swift, B.S. Andereck

\section{To cite this version:}

J. Swift, B.S. Andereck. Statics and dynamics near the nematic-columnar phase transition in liquid crystals. Journal de Physique Lettres, 1982, 43 (12), pp.437-440. 10.1051/jphyslet:019820043012043700 . jpa-00232073

\section{HAL Id: jpa-00232073 https://hal.science/jpa-00232073}

Submitted on 1 Jan 1982

HAL is a multi-disciplinary open access archive for the deposit and dissemination of scientific research documents, whether they are published or not. The documents may come from teaching and research institutions in France or abroad, or from public or private research centers.
L'archive ouverte pluridisciplinaire HAL, est destinée au dépôt et à la diffusion de documents scientifiques de niveau recherche, publiés ou non, émanant des établissements d'enseignement et de recherche français ou étrangers, des laboratoires publics ou privés. 
Classification

Physics Abstracts

$64.70 \mathrm{E}$

\title{
Statics and dynamics near the nematic-columnar phase transition in liquid crystals
}

\author{
J. Swift and B. S. Andereck \\ Department of Physics, The University of Texas at Austin, Austin, Texas 78712, U.S.A.
}

(Reçu le 11 mars 1982, accepté le 22 avril 1982)

\begin{abstract}
Résumé. - Un Hamiltonien de Landau-Ginzburg décrivant un cristal liquide nématique près de la transition nématique-phase colonne est utilisé pour calculer la renormalisation des constantes élastiques et des viscosités dans la phase nématique. Nous trouvons que les constantes élastiques, sauf la constante élastique de flexion, sont augmentées par les fluctuations du paramètre d'ordre et que cette amplification est proportionnelle au carré de la longueur de corrélation. Une des viscosités seulement n'est pas amplifiée près de la transition.
\end{abstract}

\begin{abstract}
A Landau-Ginzburg Hamiltonian describing a liquid crystal in the nematic phase near the nematic-columnar phase transition is used to find the renormalized nematic elastic constants and viscosities. The elastic constants, with the exception of the bend elastic constant, are found to be enhanced by order parameter fluctuations, the enhancement being proportional to the second power of a correlation length. Only one of the viscosities is not enhanced near the transition.
\end{abstract}

The long-range stability of the two-dimensional crystal in a three-dimensional space was demonstrated by Landau and Lifshitz [1] long before a physical realization was discovered by Chandrasekhar, Sadashiva, and Suresh [2] in a thermotropic liquid crystal system. This phase, with long-range order in two directions (a triangular lattice) and fluid properties in the third direction is known by a variety of labels (tubular, disk-like, canonic, calamitic, discotic, hexagonal), but we shall adopt Prost's [3] use of the name columnar phase. The hydrodynamics and elastic properties of columnar liquid crystals were discussed by Prost and Clark [4]. Kats [5] described the free energy and behaviour of the elastic constants near the columnar-crystalline transition and also near the nematic-columnar (N-Col) transition. As we describe our theory we will comment on some differences between our work and that of Kats. In this letter we consider the N-Col phase transition and the enhancement of the elastic constants and viscosities in the nematic phase due to order parameter fluctuations near the transition.

For liquid crystals of the type which can form a columnar phase the director is perpendicular to the disk-like molecules which comprise the liquid crystal. In the nematic phase the average orientation of the director is non-zero, i.e. the disks generally face in a common direction. At the N-Col transition we have the onset of a two-dimensional, triangular lattice perpendicular to the mean director orientation. The long-range density correlations of the columnar phase can be 
represented by choosing an order parameter analogous to the NAC-model of Chen and Lubensky (CL) [6]. The N-Col order parameter is, then

$$
m(\mathbf{r})=\int_{\mathbf{D}} \frac{\mathrm{d}^{3} k}{(2 \pi)^{3}} \mathrm{e}^{i \mathbf{k} . \mathbf{r}} \rho(\mathbf{k}),
$$

where $\rho(\mathbf{k})$ is the Fourier-transformed density and the wave vector integration is over a domain $\mathrm{D}$ which is a torus of minor radius $\Lambda$ and major radius $q_{\perp 0}$, where $q_{\perp 0}$ is the magnitude of the smallest reciprocal lattice vector of the triangular lattice $\left(\Lambda^{\circ}<q_{\perp 0}\right)$. The torus is centred at the origin and its major axis is parallel to the mean director. The choice for order parameter establishes no preferred direction in the plane perpendicular to the director.

Our Landau-Ginzburg Hamiltonian $(H)$ is very similar to that of CL but with obvious changes so that density waves perpendicular to the director are preferred. Our $H$ is written in the form

$$
\beta H=\beta H_{m}+\beta H_{\mathrm{el}}+\beta H_{3}+\beta H_{4}
$$

where the first term in $\beta H$ is

$$
\beta H_{m}=\frac{1}{2} \int \mathrm{d}^{3} r\left\{a m^{2}-C_{\|}(\mathbf{n} . \nabla m)^{2}+C_{\perp} \sum_{i j} \delta_{i j}^{T}\left(\nabla_{i} m\right)\left(\nabla_{j} m\right)+\frac{C_{\perp}^{2}}{4 D_{\perp}} m^{2}+D_{\perp}\left(\nabla_{\perp}^{2} m\right)^{2}\right\},
$$

where $\mathrm{n}$ is the director, $\delta_{i j}^{T}=\delta_{i j}-n_{i} n_{j}, a=a^{\prime}\left(T-T_{\mathrm{c}}\right) / T_{\mathrm{c}},\left(a^{\prime}\right.$ being a positive constant and $T_{\mathrm{c}}$ the N-Col transition temperature),

$$
q_{\perp 0}^{2}=-C_{\perp} / 2 D_{\perp}
$$

and $C_{\perp}<0$ and $C_{\|}<0$.

Equation (2) can be written in terms of the spatial Fourier transform of $m$,

$$
m(\mathbf{q})=\int \mathrm{d}^{3} r \mathrm{e}^{-i \mathbf{q} \cdot \mathbf{r}} m(\mathbf{r}) .
$$

If we neglect fluctuations in the director in (2) then we have

$$
\left.\beta H_{m}\right|_{\mathbf{n}=\mathbf{n}_{0}}=\frac{1}{2} \int \frac{\mathrm{d}^{3} q}{(2 \pi)^{3}}\left[a-C_{\|} q_{\|}^{2}+D_{\perp}\left(q_{\perp}^{2}-q_{\perp 0}^{2}\right)^{2}\right]|m(\mathbf{q})|^{2}
$$

where $q_{\|}$is the component of $\mathbf{q}$ parallel to the director, $q_{\perp}$ is the magnitude of the component of $\mathbf{q}$ perpendicular to the director, and $\mathbf{n}_{\mathbf{0}}$ is the equilibrium director. Obviously, in this model density waves in the plane perpendicular to the average director and with magnitude $q_{\perp}=q_{\perp 0}$ are favoured.

Note that our Hamiltonian (3) reflects the symmetry of the nematic state and that order parameter fluctuations associated with any wave vector $q$ pointing to the ring defined by $q_{\|}=0$, $q_{\perp}=q_{\perp 0}$ are equally likely $[6,7,8]$. This means that the strong X-ray scattering from order parameter fluctuations would be associated with this single ring in wave vector space.

On the contrary, Kats has modified the free energy of Jähnig and Brochard [9] and even in the nematic phase apparently assumes a definite orientation for the reciprocal lattice vectors in the plane perpendicular to the average director. Since no such preferred direction exists in the symmetry of the nematic phase we assume that he has implicitly imposed an external field or some sort of restricting boundary conditions. 
In equation (1) $\beta H_{\mathrm{el}}$ is the Frank free energy and is exactly given by equation (2.4) in $\mathrm{CL}, \beta \mathrm{H}_{4}$ is given by equation (2.5) in their paper, and, since a cubic invariant exists for our case, we have

$$
\beta H_{3}=v \int \mathrm{d}^{3} r m^{3}(\mathbf{r})
$$

where $v$ is a constant. Our calculation is a direct parallel of that of CL and assumes that the transition is not strongly first order so that the fluctuations are observable.

In equation (2), $\beta H_{m}$ may be expanded out in terms of the deviation of the director from its average value. This leads to couplings between the order parameter and director of the form given by equations (3.4) and (3.5) in CL. The vertex $\Gamma_{i}(\mathbf{k}, \mathbf{q})$ is given by their equation (3.5) but with $D_{\|}=0$ in this expression. Their formalism for the self energy of the director propagator may then be used to calculate the enhancements of the elastic constants near the N-Col transition. We find for the enhancements

$$
\begin{aligned}
& \delta K_{1}=\frac{k_{\mathrm{B}} T}{128 \pi} \frac{C_{\perp}^{2}}{D_{\perp}^{3 / 2}}\left(-C_{\|}\right)^{1 / 2} \frac{1}{a}=\frac{k_{\mathrm{B}} T}{64} q_{\perp 0}^{3} \xi_{\perp} \xi_{\|}, \\
& \delta K_{2}=\frac{k_{\mathrm{B}} T}{384 \pi} \frac{C_{\perp}^{2}}{D_{\perp}^{3 / 2}}\left(-C_{\|}\right)^{1 / 2} \frac{1}{a}=\frac{k_{\mathrm{B}} T}{192} q_{\perp 0}^{3} \xi_{\perp} \xi_{\|},
\end{aligned}
$$

and

$$
\delta K_{3}=0
$$

Our enhancement for the splay elastic constant is proportional to the square of a correlation length whereas Kats' result is linear in a correlation length. The difference stems from the integration regions used in calculating the free energy. Kats' preferred direction for the reciprocal lattice vector $\mathbf{p}_{0}$ gives him a small three-dimensional spherical integration region around $\mathbf{p}_{0}$, making his problem most similar to the nematic-smectic-A problem. Our expression for the free energy involves no unique direction in the plane of the triangular lattice and hence requires a toroidal integration region, similar to the nematic-smectic- $\mathrm{C}$ case. According to reference [7] the high degree of degeneracy in this case effectively lowers the dimensionality of the wave vector integration from three to two and leads to an additional factor of $\xi$, as was seen in CL's results. We find similar enhancement for the twist elastic constant (as seen in Eq. (4b)). Kats states that his formulation only gives an increase in $K_{1}$.

The enhancements of the viscosities near the N-Col transition are found by techniques paralleling Hossain, Swift, Chen and Lubensky [11]. Again corrections to the various propagators (associated with the director and velocity) are calculated. The inverse propagators are not readily separable, but form a matrix in which the simple mode coupling fluctuation corrections can be included and from which the perturbed values of the viscosities can be found. For a sketch of the procedures involved, see the Appendix of reference [10]. Enhancements are again found [11]. In the notation of reference [10] the enhancement of the " viscosity " associated with the director is

$$
\gamma_{1} \equiv \gamma_{1}^{0}+\tilde{\gamma}_{1}=\gamma_{1}^{0}+k_{\mathrm{B}} T \frac{\gamma_{3}^{0}}{64 \pi} \frac{\left(-C_{\|}\right)^{1 / 2}}{D_{\perp}^{1 / 2}} q_{\perp 0}^{2} \frac{1}{a}=\gamma_{1}^{0}+k_{\mathrm{B}} T \frac{\gamma^{0}}{128 \pi} \frac{q_{\perp 0}}{D_{\perp}} \xi_{\|} \xi_{\perp}
$$

and the corrections to the nematic liquid crystal viscosities are

$v_{1}=v_{1}^{0}+k_{\mathrm{B}} T \frac{\gamma_{3}^{0}}{256 \pi} \frac{1}{\left(-C_{\|}\right)^{1 / 2} D_{\perp}^{1 / 2}}\left(-C_{\perp}\right) q_{\perp 0}^{2} \frac{1}{a}=v_{1}^{0}+k_{\mathrm{B}} T \frac{\gamma_{3}^{0}}{512 \pi} \frac{q_{\perp 0}^{2}}{\left(-C_{\|}\right)^{1 / 2} D_{\perp}^{1 / 2}} \xi_{\perp}^{2}$ 
$v_{2}=v_{2}^{0}+k_{\mathrm{B}} T \frac{\gamma_{3}^{0}}{128 \pi} \frac{1}{\left(-C_{\|}\right)^{1 / 2} D_{\perp}^{1 / 2}}\left(-C_{\perp}\right) q_{\perp 0}^{2} \frac{1}{a}=v_{2}^{0}+k_{\mathrm{B}} T \frac{\gamma_{3}^{0}}{256 \pi} \frac{q_{\perp 0}^{2}}{\left(-C_{\|}\right)^{1 / 2} D_{\perp}^{1 / 2}} \xi_{\perp}^{2}$

$v_{3}=v_{3}^{0}+k_{\mathrm{B}} T \frac{\gamma_{3}^{0}}{32 \pi} \frac{D_{\perp}^{1 / 2}}{\left(-C_{\|}\right)^{3 / 2}} q_{\perp 0}^{2} \ln \frac{\Lambda^{2}}{a}$

$v_{4}=v_{4}^{0}+k_{\mathrm{B}} T \frac{\gamma_{3}^{0}}{64 \pi} \frac{1}{\left(-C_{\|}\right)^{1 / 2} D_{\perp}^{1 / 2}}\left(-C_{\perp}\right) q_{\perp 0}^{2} \frac{1}{a}=v_{4}^{0}+k_{\mathrm{B}} T \frac{\gamma_{3}^{0}}{128 \pi} \frac{q_{\perp 0}^{2}}{\left(-C_{\|}\right)^{1 / 2} D_{\perp}^{1 / 2}} \xi_{\perp}^{2}$

$v_{5}=v_{5}^{0}$.

In addition, there is a correction to the reactive coefficient $\lambda$ given by $\lambda=\left(\gamma_{1}\right)^{-1}\left(\lambda^{0} \gamma_{1}^{0}+\tilde{\lambda} \tilde{\gamma}_{1}\right)$ and near the N-Col transition $\tilde{\lambda}=1$.

We have found enhancements due to order parameter fluctuations for all but $K_{33}$ and $v_{5}$ as the $\mathrm{N}-\mathrm{Col}$ transition is approached in the nematic phase. The critical behaviour of elastic constants and viscosities can be studied experimentally by measuring the speed and attenuation of sound. Such measurements would provide a test of the assumption that the N-Col transition can be treated as nearly second order and could also be a check on the accepted symmetry of the columnar phase.

Acknowledgments. - This work was supported by the Robert A. Welch Foundation through Grant No. F-767.

\section{References}

[1] Landau, L. and Lifshitz, E. M., Statistical Physics (Addison-Wesley, Reading Mass.) 1969, p. 402.

[2] Chandrasekhar, S., Sadashiva, B. K. and Suresh, K. A., Pramana 9 (1977) 471.

[3] Prost, J., preprint.

[4] Prost, J. and Clark, N. A., Bangalore Int. Liq. Cryst. Conf. (Addison-Wesley) 1980.

[5] Kats, E. I., Sov. Phys. JETP 48 (1978) 916.

[6] Chen, J. and Lubensky, T. C., Phys. Rev. A 14 (1976) 1202.

[7] Swift, J., Phys. Rev. A 14 (1976) 2774.

[8] AleXander, S. and MCTAGUe, J., Phys. Rev. Lett. 41 (1978) 702.

[9] JÄhNIG, F. and BroChard, F., J. Physique 35 (1974) 301.

[10] Hossain, K. A., Swift, J., Chen, J., Lubensky, T. C., Phys. Rev. B 19 (1979) 432.

[11] We find, for the N-Col transition, in the notation of reference [9] :

$$
\begin{aligned}
& \alpha=\gamma_{3}^{0}\left(-C_{\|}\right)^{1 / 2} D_{\perp}^{-1 / 2} q_{\perp 0}^{2} / 64 \pi a \\
& \beta=\gamma_{3}^{0}\left(-C_{\|}\right)^{3 / 2} D_{\perp}^{1 / 2} q_{\perp 0}^{2} \ln \left(\Lambda a^{-1 / 2}\right) / 32 \pi \\
& \Delta=\gamma_{3}^{0}\left(-C_{\|}\right)^{-1 / 2} D_{\perp}^{1 / 2} q_{\perp 0}^{4} / 64 \pi a .
\end{aligned}
$$

We find that $\Phi$ and $\sigma$ have a logarithmic dependence on $a$, but their contributions are dominated by $\Delta$. 